cases, without exception, there was diminution or absence of the vestibular irritability. In three cases there was negative irritability on both sides; in one case (58) there was negative irritability on the one side and very much diminished on the other. In two cases there was much diminished irritability on both sides. In one case there was negative irritablity on the one side and probably normal on the other, but this case (54) was complicated by strong ocular nystagmus which made the examination difficult. In none of the cases was there any discomfort or vertigo produced by the syringing with cold water, nor was there any disturbance of equilibrium. The results in these cases of congenital syphilis is important and interesting. That there was such a constant obliteration of the labyrinth was up till now not known.

XIII. (One case, 61.) The diagnosis of chronic adhesive process with lesion of the internal ear was made. In this case the restibular irritability was + .

XIV. (Fourteen casts, 61-69, 71-75.) Cases with the character of a lesion of the internal ear without discoverable cause. Of these cases:

9 had the vestibular irritability normal.

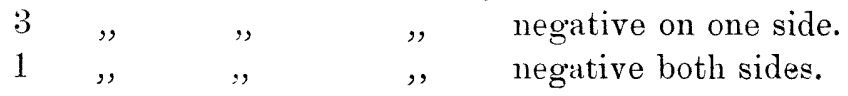

\title{
ANTRAL DISEASE IN RELATION TO SPECIAL AND GENERAL SURGERY.1
}

\author{
By Herbert Thlley, M.D.Lond., F.R.C.S.Exa., \\ Surgeon, Ear and Throat Department, University College Hospital.
}

THE diseases of the maxillary antrum appeal to the general as well as the special surgeon, and the latter comprise the rhinologist or surgeon with special knowledge of nasal diseases, and the dental surgeon whose studies have been focussed on pathological conditions of the teeth. Speaking generally, it may be said that the general surgeon will be more familiar with those diseases which cause obvious external swelling or deformity of the upper jaw or of the adjacent soft parts, and so he will be consulted for large alveolar abscesses, dental cysts, malignant disease of the antrum, and necrosis of the upper jaw. Since these affections are

\footnotetext{
1 Read in the Section of Dental Surgery of the British Medical Association, by kind permission of the Editors.
} 
also frequently seen by the rhinologist and dental surgeon, we we may as well discuss them at once.

Alveolar Abscess. - When an abscess forms at the root of one of the incisor teeth the swelling may appear in the floor of the nose, just within or beyond the vestibule, and cause nasal obstruction of a marked degree. When the canine tooth is affected the tumefaction may appear externally at the side of the nose below the nasal bone, and may seem at first sight to have little to do with a diseased tooth. If such abscesses become chronic, cholesterin crystals are often found in their contents. Treatment must be directed to removal of the diseased tooth and opening the alveolar abscess.

Dental Cysts.-These, by their expansion, often cause considerable external deformity, and may so encroach upon the antral cavity that the sinus is almost obliterated. Pain, as a rule, is slight or absent, the swelling over the anterior antral wall within the mouth frequently presents the so-called "egg-shell crackling" when pressed upon, the hard palate is never pressed downwards, and if the transillumination test be applied, the affected antrum is only slightly less translucent than the normal one, or at any rate it is not quite opaque. To be successful treatment must be radical, for nothing is more wearisome to surgeon and patient than the constant packing of the cyst cavity after it has been merely opened from the mouth. The most effectual, speedy, and certain treatment is to reflect the mucous membrane from the buccal aspect of the cyst wall, freely open the cyst, remove all its thin, bony wall, especially that portion which projects into, and often almost entirely obliterates the true antral cavity, then remove the inner antral wall together with the anterior half of the inferior turbinal, so that a large permanent opening into the nasal cavity is provided. Finally, the bucco-antral incision is sutured with a couple of horsehair stitches, and immediate union of the wound will take place. Practically there is no after-treatment beyond the use of a warm alkaline nasal douche for two or three weeks.

Malignant Disease of the Antrum.-Sarcoma and epithelioma are the commonest forms of malignant disease which affect the antrum. Pain of an intense and boring character is often an early symptom in epithelioma. With increase of the growth there may be distension of the anterior antral wall, producing swelling of the cheek, depression of the hard palate, nasal obstruction and bleeding from the nose. Epiphora is a not uncommon symptom, and is due to the invasion of the tear duct by the growth. Trans- 
illumination reveals marked opacity of the antrum, and exploration of the antrum through the inferior meatus shows no sign of pus, but is often followed by the How of a few drops of blood through the canuula. 'The only efficient treatment is a free excision of the upper jaw, and the operation should be performed directly the diagnosis has been made. Care must be taken not to mistake a large cyst for malignant disease of the antrum, and the following points will enable a diagnosis to be made:

$$
\text { Cyst. }
$$

Pain slight or absent.

Transillumination more or less Quite opaque. clear.

Fluid contents on exploration. No discharge, or only a few drops of blood.

Necrosis of the Lpper Jau incolving the Antwum.-This is usually the result of tertiary syphilis. There may be slight swelling or cedema of the cheek, with obliteration of the folds between the ala nasi and the cheek. Frequently there is a foul discharge from the corresponding nostril, and careful intra-nasal examination may enable the surgeon to detect sequestra on the outer wall of the nasal cavity. The diagnosis will depend on the above signs, together with a history of syphilis and the presence of luetic lesions in other parts of the body. The treatment must be constitutional as well as local, and, with regard to the latter, general narcosis may be necessary to remove loose sequestra inaccessible by way of the nasal passages.

\section{Antral Suppuration.}

We may now pass to the acute and chronic inflammatory lesions of the antrum, which are of special interest to the rhinologist and dental surgeon, and let me say at once that I propose to discuss antral suppuration only as we find it when limited to that sinus alone, for I scarcely think it would be fitting if, in this section, we included in one purview those cases which come before the nasal surgeon in which the antral and other sinuses are simultaneously involved.

Before discussing the causative factors of antral inflammation let me point out one or two anatomical features of the antrum which may be of surgical importance, and which should influence us in the treatment which we adopt for the relief of intlammatory 
lesions. The exigencies of time compel me to assume that you are all familiar with the normal anatomy and development of the maxillary sinus.

(1) The antral cavities often vary much in size in the same individual, and I can show you a beautiful stereoscopic picture which illustrates this point.

(2) When there is a tendency for the palate to be high and the face is of the narrow type, then the alveolar process will be thick and the antrum relatively small-a type more common in women than in men, in whom the converse condition of things is more generally true. It will be obvious how difficult it will be to efficiently drain the antrum by way of the alveolar route in the first type of case.

(3) The inner antral wall is of interest in comnection with the more radical operative measures. Regarded from the antral side, this wall is divided into two parts by the line of attachment of the inferior turbinal; the anterior and lower triangle is bony, and corresponds to the inferior meatus; the upper and posterior is mainly membranous, and forms a portion of the middle meatus, and in it is situated the natural ostium of the antrum.

(4) The actual cavity of the antrum is often very irregular, and may be more or less divided up by bony septa. Irregular depressions are frequently present over the roots of the teeth which approximate to the sinus cavity, and, in addition, there are often to be met with the pre-lacrymal and palatine recesses, illustrations of which $\mathrm{I}$ am able to show you. Your attention is drawu to these prints because in these pockets infection may long be harboured, and only some form of radical procedure can be expected to deal successfully with them.

(5) Finally, certain of the lower anterior ethmoidal cells may extend externally into the imner, posterior, and superior regions of the roof of the antrum. These are known as maxillo-ethmoidal cells, and may be the cause of a chronic antral suppuration, and can only be dealt with by some form of radical operation.

It is now a well-established fact that infection of the antrum may arise by way of the nasal cavities or through disease of certain teeth. Acute antral suppuration by the intra-nasal route is most commonly due to infection by the pathogenic organisms of certain of the acute specific diseases. Of these influenza has been the most active, although the worst case of bilateral suppuration of all the nasal accessory sinuses which I have seen followed enteric fever, and one of the most interesting was met with in a lad, aged 
uine, where acute frontal sinus suppuration, necessitating external operation, complicated scarlet fever. Many cases recover spontaneously, and failure to achieve the desired end is often due to coincident pathological conditions within the nose, whereby free and spontaneous drainage is hindered. It is not an infrequent. esperience to meet with bilateral acute antral suppuration in which one side recovers spontaneously, but owing to the presence of a large septal spur or deviation on the other side drainage has been impeded, and the acute has passed into the chronic stage of suppuration.

A second common cause of antral suppuration is infection from a diseased tooth, an apical abscess, or suppurative periodontitis. Less frequent retiological factors are traumatisms, dental as well at intra-nasal, and amongst these we camnot overlook unskilful t'xtractions, as well as the careless nse of the galvanic cautery within the nose.

Chronic suppuration within the autrum is usually the sequela of an acute attack of inflammation which has failed to undergo resolution. Such failure may be due to the virulence of the initial inflammation, to defective drainage caused by pathological intranasal conditions, or to the continuance of the initial cause of the inflammation-for example, an apical dental abscess which continues to supply infective material to the inside of the antrum. And finally, a debilitated state of the patient's general health will be a strung predisposing factor in the lormation and contiuuance of chronic suppuration in one or more of the sinuses.

As regards the bacteriology of the condition, time will not allow me to go into this interesting matter, and those who desire informatirn on the subject I would refer to the work carried out by Drs. Ligan 'l'urner and Lewis ${ }^{1}$ who, amongst other conclusions, have shown "that the pus obtained from some cases of antral suppuration may coutain organisms similar to those occurring in the buccal ("ivity; that occasionally bacilli distinctive of dental caries may be isolated from the pus of an antral abscess; that in the cases of chronic suppuration streptococei were found in 80 per cent., whilst in the more recent cases they occurred in 60 per cent.; that in recent cases virulent organisms are met with twice as often as in cases of chronic suppuration; that clinical evidence supports the view that the antrum is more frequently infected by way of the nasal cavity, and that this opinion is corroborated by bacteriolugical investigation."

1 Edin. MLed. Journ., 1905. 


\section{Accte Antral Suppuration.}

Symptoms.-When the inflammation is the result of dental infection the earlier symptoms are usually located around the tooth, and these are so well known to you that I need not waste time in describing them. 'Their relief is often coincident with a foul nasal discharge, which intra-nasal examination would probably reveal as it issued from the middle meatus between the lower border of the middle and the upper border of the inferior turbinal.

Prognosis.-This will be particularly good if the offending tooth be removed early and suitable treatment be instituted.

Treatment.-When antral suppuration is obviously of dental origin, the offending tooth should be removed and free communication with the antrum made through the alveolus by means of a suitable perforator. 'The sinus should be gently irrigated with a warm sterile normal saline solution, boracic lotion, weak Condy's fluid, or with any one of the numerous mild fragrant antiseptics which are now on the market. The alveolar opening should be kept open by a suitable plug, and irrigation practised twice or thrice daily at first, aud at increasing intervals as the amount of pus observed in the returning fluid becomes less. When, after an interval of five to seven days, no pus is seen in the basin after irrigation, the plug may be finally removed overnight, and by the morning the alveolar perforation will be nearly closed. When antral suppuration is of nasal origin the prognosis is less favourable, and the treatment may be less simple, for reasons which will be discussed immediately. Under these circumstances we must endeavour :

(1) To treat the primary constitutional disease by rest in bed, suitable food, and such internal medication as the nature of the general infection may indicate.

(2) To allay the local discomfort caused by the complicating acute sinusitis. The neuralgic pain will frequently be relieved by 10-grain doses of aspirin every four or six hours, and hot fomentations to the cheek may assist to the same end.

(3) But locally our chief efforts must be directed to promoting the free and spontaneous discharge of pus from the antrum by way of the natural ostium, and these ends may be attained:

(a) By directing the patient to lie in bed with the diseased antrum uppermost.

(b) The application of cocaine and adrenalin solutions to the 
regions around the middle meatus. This may be done every four or six hours.

(c) Scarification of these regions is recommended by Lack, and my experience confirms the efficacy of the method.

(d) In the intervals of such treatment inhalation of mentholised steam will have a similar effect in inducing contraction of the swollen intra-nasal mucosa.

If these means fail, the antrum should be punctured through its inner wall in the inferior meatus and irrigated with warm, mild antiseptic lotions. If this be done daily or on alternate days for five or six times, resolution will generally take place. Should suppuration continue, then a larger intra-nasal opening must be made, and this will practically always succeed in curing acute antral suppuration for which milder measures have failed. It will thus be seen that the clinical course, prognosis, and treatment of acute suppuration of dental origin are more favourable than when the sinus is infected by way of the nose. In the one case we have a small local source of infection, the greater part of the mucous membrane of the antrum and its corresponding nasal cavity being practically healthy; in the other (intra-nasal infection) the whole mucous membrane of the nose and the antrum seems to be intimately infected and inflamed, while the resisting power of the patient is frequently reduced to a low point by the general infection of the system.

\section{Chronic Antral Suppcration.}

We have already said that chronic antral suppuration is the sequel of an acute dental or intra-nasal infection, which for some reason or reasons has not undergone resolution.

Symptoms. - Whatever be the original cause of the trouble, the patient will usually complain of one or more of the following symptoms: (a) An unpleasant odour in the nose, which is often most marked on lowering the head. (b) Headache, often over the corresponding eye, and the aching may have a marked periodicity - for example, it is peculiarly apt to occur in the morning, and pass off about midday - and this periodicity has often led to it: being mistaken for a malarial symptom, especially when the patient has resided in regions where the disease is endemic. (c) More or less nasal obstruction, which will be caused by polypi or abnormal swelling and congestion of the nasal mucous membrane. Cough, liability to colds in the head, anæmia, indigestion, anorexia, and 
general debility are often complained of, and in the case of the last four symptoms they may be due to the constant swallowing of the foul discharge. Not infrequently the absorption of certain of the toxins into the system exerts a curious effect on the nervons system, so that the patient becomes depressed, apathetic, ant incapable of any prolonged mental exertion.

Diagnosis.-In a suspected case of chronic antral suppuration our first duty will be to examine the teeth on the same side, ant also the corresponding vasal cavity. I need scarcely warn dental surgeons that a tooth which does not ache is not necessarily and de facto guileless; and furthermore, that the secret of an antral suppuration often is more obvious underneath than on the surface of a gold crown. Intra-nasal examination will often reveal pus in the middle meatus, but, if this sign be absent, it may sometimes: be induced by asking the patient to turn the suspected cheek towards the ceiling for a few moments, when the discharge may be caused to flow into the middle meatus. Transillumination is a very valuable diagnostic help, if used with discretion and if taken in conjunction with the other symptoms exhibited by the patient. The 10-volt. lamp is placed in the patient's mouth, his lips tightly closed, and the room darkened, or the surgeon and patient's head enveloped in a dark cloth. If the antrum be healthy, a crescent of light is seen in the infra-orbital region; if the antrum br inflamed, the region referred to will be dark and in marker! contrast with the healthy side. It should emphatically be statel that a dark antrum does not necessarily mean antral suppuration, but, on the other hand, good transillumination may be taken to exclude that condition. Before applying the test you must remor any denture from the upper jaw, or the antrum may be quite opaque. I once saw an antrum opened because this little point in diagnosihad been neglected.

Exploration Test.-This is a final and certain test when other means fail, as they frequently do. A fine trocar and cannula are passed through the inner wall of the antrum underneath the inferior turbinal. Some warm normal saline solution is then injected into the antrum and returns from the nose; the presence of pus in the returning fluid can thus be determined with absolute certainty. The little operation is almost painless, and its evidence is absolutely reliable. Some surgeons make the puncture in the middle meatal region through the membranous part of the inner antral wall, and claim that it is easier and less painful than beneath the inferior turbinal. (The instrument aud method of 
using it were demonstrated.) I have frequently found that perforation and irrigation of the antrum have been immediately followed by pain in a certain diseased tooth, and this affords a valuable hint as to which of two teeth possibly was the real offender.

Prognosis.-As in the acute inflammations, chronic suppuration of dental origin is of better prognosis than when due to intra-nasal infection, and for the reasons already given.

Treatment.-In discussing the treatment of a chronic antral empyema, we must remember that we are dealing with an abscess of a bony cavity the walls of which cannot contract, and that if we are to check the suppuration and promote the return of the mucous membrane to a natural condition we must provide for free, unhindered, spontaneous drainage. These ends may be secured by drainage through the alveolus, from the cannine fossa, or by an opening from the antrum into the nose. The principles which should guide us in the selection of the method of operation is the main olject of my theme to-day, and it is upon the method of treatment that discussion will probably centre, and differences in opinion based upon experience will be as numerous as they are valuable.

Alceolar Drainage. - If the clinical history seems to show that the antral suppuration was of dental origin, and examination has proved that the second bicuspid or the first or second molar teeth are diseased, then it may reasonably be argued that such a tooth or teeth should be removed, a free communication made with the intrum, a plug or tube inserted, and frequent irrigation practised until the discharge lessens or completely ceases. This method will undoubtedly be successful in a certain number of cases, and especially in those where the antral suppuration has been maintained by an apical abscess or suppurative periodontitis. In such instances the greater part of the autral mucous membrane is healthy, the infection is more or less local, and only calls for removal of the infecting focus.

Such cases as these frequently and uaturally come under treatment by the dental surgeon, and the result is often so quickly successful that he is enamoured of the alveolar route, and considers it more universally applicable than would be the case if he had an equal experience of empyemata caused by other atiological factors. For example, a foetid, purulent nasal discharge frequently leads the patient to consuit a rhinologist, and it may be the work of a few moments to determine the presence of an antral abscess. He tells us that the symptoms date from a severe cold, or influenza, 
possibly three or four months previously. Examination of the teeth may reveal exteusive caries of the bicuspids or molarspossibly one of these may be "dead." The tooth is removed, the alveolus perforated, a plug inserted, and irrigation practised. In spite of change of lotion, change of air, and possibly change of doctor, the suppuration continues, although its foetor may be destroyed, and its amount may be diminished; still, the fact remains, the daily irrigation has to be continued, because its cessation means a return of the unpleasant symptoms. Why is this so? Why is the treatment which was so conspicuous by success in the first case a failure in the second?

Possibly the answer has already been given-namely, that in those cases due to intra-nasal infection there is a more general and intimate inflammation of the antral mucous membrane, that the dental disease is scarcely more than a coincidence, and that the alveolar puncture, even though it might admit the passage of a slate pencil, does not provide for free, unhindered, spontaneous drainage; and, futhermore, it provides an additional source of infection by way of the mouth. In this consideration the nature and virulence of the infecting organisms may play a conspicuous part in promoting chronicity, and those who have made a special study of the bacteriology of antral discharges may be able to throw some light on this point.

In my own practice, as well as in those of my colleagues and of dental surgeons, I have seen a large number of failures with alveolar drainage. The causes of some of these have been very obvious: For example, (1) drainage-tubes which projected half an inch above the level of the antral floor, and therefore did not drain until the antrum was already half filled with pus ; (2) drainagetubes of narrow calibre, which do no drain at all, because mucus and pus almost immediately coagulate and obstruct their lumen when they are inserted; (3) tubes which are too short, and therefore the upper part of the alveolar tract becomes closed by granulation tissue, which has to be broken down every time irrigation is practised; (4) tubes which have a free opening into the mouth, and therefore may admit food and other infective elements, which act deleteriously on the antrum.

I have often admired the mechanical skill and ingenuity expended on antral plugs and tubes and regretted that the result obtained by their use was not equally worthy of congratulation, and the consequence has been that in my own practice, when 1 utilise the alveolar route, which is very seldom, I insert a small 
vulcanite plug with a milled shank, which is easily retained. Truly this does not provide for drainage from the most dependent position of the antrnm, but it provides and keeps open a passage which can be used for irrigation as often as necessary, and in the intervals access from the mouth to the sinus is closed. We may say, then, of alveolar drainage that it will probably be successful in all acute cases of dental origin and in a moderate proportion of chronic cases due to the same retiological factor. A plug is probably more satisfactory than a tube, because, unless the calibre of the latter is comparatively large, drainage does not take place, and the antral cavity may be contaminated by infection from the mouth.

Drainage throngh the Canine Fossu-By this method a large opening is made in the canine fossa, the soft parts and periosteum having been first reflected from the bone. Through the opening the mucous membrane may be curetted, and the buccoantral opening is kept open by rubber plugs, wicks of gauze, etc. One only mentions this operation to condemn it, and anyone who has ever employed it will have vivid recollections of the difficulties involved in keeping the opening patent, of the pain caused by socalled self-retaining plugs, which usually manage to find entry into and hide themselves in the antrum, of the difficulties of irrigation, and, above all, of the failure of such a method to cure.

Intra-nasal Drainage.-The advantages of treating a chronic antral suppuration by this route are numerous, and extended experience has proved beyond cavil or dispute that this method is the best for all cases where the primary infection has been by way of the nose, and for those instances of chronic empyemata of undoubted dental origin which have failed to get well when drained by the alveolar route, or where there are reasons for not adopting that method.

Intra-nasal drainage may be established by two methods :

(1) The Calduell-Luc method, in which a large opening is first made in the canine fossa, and then the greater part of the inner or naso-antral wall is removed, including the anterior half of the inferior turbinal. When any diseased portions of antral mucous membrane have been removed and bleeding has ceased, the buccoantral wound is sutured, and usually heals by immediate union. No packing should be inserted into the sinus, and the aftertreatment consists of intra-nasal irrigation of two to four weeks by way of the nose. Few operations give such excellent, speedy, and permanent results, but in order to ensure success we must- 
(a) Make a large opening into the vose-and I am in the habit of removing practically all the naso-antral wall, because small openings have a great tendency to contract.

(b) Not curette away the healthy mucous membrane of the sinus. It is surprising how swollen, œdematous, and apparently degenerate mucous membrane will recover itself if free, unhindered, spontaneous intra-nasal drainage be provided.

(2) The Simple Intra-nasal Route.-In this method the anterior half of the inferior turbinal is removed and then, by means of specially-constructed burrs, trocars, or forceps, the inner antral wall, in whole or in part, is removed, until an opening is made which will easily admit the tip of the little finger. 'This method is simpler than the Caldwell-Luc operation, and has recently been much advocated. During the past twelve months I have frequently employed it, and am very satisfied with the results which it gives in selected cases. It is especially indicated in chronic cases of dental origin, when the anterior ethmoidal region shows no signs of infection, or when the middle meatal region is free from polypus formation. On a few occasions I have made use of the operation for acute empyema of nasal origin, in which alveolar puncture has not relieved pain or diminished the discharge. Its advantages over the Caldwell-Luc operation are :

(a) It involves far less traumatism of the parts, and may therefore be the chosen operation for the old, weak, or infirm.

(b) It is a much simpler operation, and may be thoroughly performed in about five minutes.

Its great disadvantage is that the surgeon cannot inspect the diseased cavity upon which he is operating, and must rely on the evidence afforded by the tip of his little finger inserted through the nostril and inner antral wall. When in addition to antral suppuration polypi are present in the middle meatus, I think it wiser to carry out the more radical Caldwell-Luc operation, because more detailed treatment of the antral mucous membrane may be called for, and ready access may be obtained to the ethmoidal or maxillo-ethmoidal cells.

To sum up, we may state:

(1) The alveolar route is especially suitable for acute empyemata of dental origin; that it will cure a certain number of chronic cases due to the same cause, and the likelihood of success is greater the earlier the method is adopted. The method is not suited for cases of intra-nasal origin. A solid plug will be more suitable than a hollow one. 
(2) Intra-nasal drainage by oue or other route is a more satisfactory method for dealing with chronic antral abscess-

(a) Because free drainage into the nose is established, and this is continuous and permanent, and it involves no trouble to the patient beyond irrigation with some mild aseptic for a few weeks after the operation. Contamination from the mouth is also prevented, and the sacrifice of a useful, though possibly not a somi, tooth, will not be called for.

(b) From the patient's point of view, there are no painful afterAressings, and neuralgic pain, so frequent after alveolar puncture (so that the patient often drearls the use of the syringe), is almost unknown with the more radical procedures.

(c) Convalescence from the more radical Caldwell-Luc operation is rapid, the patient is rarely detained indoors beyond five to seven days. The simple intra-nasal operation is often done in the outpatient department of hospitals, and the patient goes home a few hours later.

With regard to the treatment of chronic antral empyemata by means of vaccines, of by treatment hased upon the opsonic index, I have not had a large experience, and the few patients who were thus treated did not give satisfactory results, although the treatment was carried out by experts.

It seemed to me that their chief difficulty laid in the fact that a variety of organisms were present in each case.

It would be interesting to know from those who have had much experience of this form of treatment whether it is satisfactory is a practical method, the length of time usually required, and finally, whether such a treatment might be useful in cases which prove obstinate by surgical methods already describerl.

It will thus be obvions to you that apart from acute cases of (1)viously dental origin, where alveolar drainage may be adopted with every hope of success, I am pleading to-day for the more frequent adoption of the intra-nasal drainage, a method which has more advantages than, and none of the drawbacks of, the alveolar. method, while its employment is in complete accord with those general principles of-surgery which should guide us whenever we endeavour to cure chronic suppuration of a bony-walled cavity. 